\title{
Expression of Phosphatase Regenerating Liver-1 in Esophageal Squamous Cell Carcinoma Associated with Metastasis
}

\author{
Yuqiong Liu, ${ }^{1}$ Xi Lou, ${ }^{2}$ Ke Guo, ${ }^{3}$ Jun-Yi Lei, ${ }^{2}$ and Huixiang Li ${ }^{1}$ \\ ${ }^{1}$ Department of Pathology, The First Affiliated Hospital, Zhengzhou University School of Medicine, Zhengzhou 450052, China \\ ${ }^{2}$ Jdxpath Laboratory, Collegeville, PA 19426-1889, USA \\ ${ }^{3}$ Institute of Molecular and Cell Biology, Proteos 138673, Singapore
}

Correspondence should be addressed to Huixiang Li, huixiang19@yahoo.com.cn

Received 24 March 2011; Accepted 20 April 2011

Academic Editors: B. Azadeh and A. Gocht

Copyright () 2011 Yuqiong Liu et al. This is an open access article distributed under the Creative Commons Attribution License, which permits unrestricted use, distribution, and reproduction in any medium, provided the original work is properly cited.

\begin{abstract}
Expression of PRL-1 protein was examined with immunohistochemistry in 60 cases of esophageal squamous cell carcinoma (ESCC) with matched lymph node metastasis $(n=40)$ and 6 cases of esophageal adenocarcinoma. Its association with ESCC metastasis was also explored. The results showed that PRL-1 protein was more frequently expressed in ESCC with metastasis $(31 / 40,77.5 \%)$ than in ESCC without metastasis $(10 / 20,50 \%, P=.031)$ and more in metastatic ESCC $(39 / 40,97.5 \%)$ than primary ESCC $(68.3 \%, P=.003)$. PRL-1 protein expression significantly correlated with the stage of ESCC, 79.4\% (27/34) in stage III ESCC and $33.3 \%(1 / 3)$ in stage 1 ESCC $(P=.003)$. PRL-1 protein was characteristically expressed in the nuclei, in addition to the cytoplasm, of 3 out of $4(66.7 \%)$ poorly differentiated esophageal adenocarcinoma. These findings suggest that PRL-1 may serve as the potential biomarker to predict the metastasis of ESCC and a better biomarker than PRL-3 in the differential diagnosis of poorly differentiated ESCC.
\end{abstract}

\section{Introduction}

Esophageal carcinoma remains one of the most common human malignant tumors worldwide [1]. In China, the incidence of esophageal carcinoma is up to $50 / 10^{5}$ with the mortality of $100 / 10^{5}[2,3]$. The effective treatment depends on early diagnosis, with which over $90 \%$ of the patients can survive 5 to 10 years [2]. Deep invasion and metastasis account for the main causes of death for the patients with esophageal carcinoma. Most esophageal carcinoma in China is squamous cell carcinoma, different from predominantly adenocarcinoma in Western countries. Nutrition, tobacco, and alcohol are believed to be the major contributors for development of esophageal squamous cell carcinoma (ESCC), whereas Barrett's esophagus is considered a key precancerous lesion with a strong association with the development of dysplasia and subsequent esophageal adenocarcinoma $[3,4]$.

Protein tyrosine phosphatases (PTPs) are a rapidly growing subgroup of protein phosphatase super family and involved in regulating functions of diverse proteins controlling numerous essential events in eukaryotes, such as transcriptional regulation, apoptosis, cell cycle progression, protein degradation, and protein trafficking [5-7]. Phosphatase of regenerating liver (PRL) is a new subclass of PTP family and has 3 members so far, PRL-1, PRL-2, and PRL-3 $[8,9]$. Although PRLs are expressed in many normal tissues at different levels $[8,10,11]$, aberrant expression of PRLs has been identified in a variety of cancer cell lines and tissues, especially in metastatic colorectal cancer [12-16]. Preliminary experiments have demonstrated that inhibition of endogenous PRL-3 in cancerous cells can abrogate cell motility and ability to metastasize in a mouse model [17]. These findings strongly suggest PRL's potential to serve as biomarkers and therapeutic targets in cancer.

The frequency of PRL-3 expression was observed in human colorectal, gastric, hepatic, ovarian, cholangiocarcinoma, nasopharyngeal, esophageal, and breast cancers as compared with their normal tissues [13-16, 18-23]. PRL-1 mRNA expression was also more often seen in the ESCC [24]. But it remains unknown in tumor for expression of PRL1 protein, which is expressed in many more normal tissues 
than PRL-3 [9-11], especially in several digestive epithelial tissues (esophagus, stomach, intestine, and liver) [25]. This study focused on the expression of PRL-1 protein in primary ESCC and their metastatic lesions in lymph node. Correlation between expression of PRL-1 proteins and clinicopathological features was analyzed.

\section{Materials and Methods}

2.1. Tissue Samples. Sixty cases of ESCC were selected from surgical pathology files in the Department of Pathology, Anyang Tumor Hospital, Henan, China. Among those 60 cases, 40 cases had matching metastatic lymph nodes and 20 had normal esophageal mucosa taken from resection margins. The diagnoses of ESCC were reconfirmed histologically for all the tumors. Tumor was classified and staged according to World Health Organization criteria [3]. Out of 60 ESCCs, 11,34 , and 15 are well, moderately, and poorly differentiated, respectively. Fifteen, 38, and 7 cases were stages I, II, and III. Six cases of esophageal adenocarcinoma (EA) were also chosen, classified, and staged in the same way. Among 6 cases of EA, 1 and 5 are well and poorly differentiated.

The patients consisted of 38 men and 22 women, and their ages ranged from 43 to 77 years old with the mean age of 61 . None of the patients received any type of therapy prior to surgery. Informed consent was obtained from all of the patients. The study was approved by the institutional review board.

2.2. Immunohistochemistry. Two to three most representative sections (slides) were selected for each case and stained with monoclonal antibody against PRL-1 (provided by Dr. KeGuo, coauthor). The procedure was as described previously in $[26,27]$. Briefly, 5-micron-thick sections were deparaffinized, incubated with pepsin (Sigma) in $37^{\circ}$ water bath for 5 minutes for antigen retrieval, and then quenched with 3\% hydrogen peroxide for 15 minutes. After blockage in 5-10\% normal goat serum for 20 minutes, the slides were stained with monoclonal antibody against PRL-1 (all in $1: 50$ dilution) at $4^{\circ}$ overnight and then incubated for 30 minutes each with goat antimouse IgG (Zymed, USA) and Streptavidin-HRP (Zymed, USA). The positive reaction was visualized with 3, 3-diamino-benzidine tetraphydrochloride (Zhongshan Golden Bridge, Beijing, China). The slides were counterstained with hematoxylin. The negative control was performed in the same way except omission of primary PRL1 antibody.

Five hundred cells from 5 representative areas of ESCC were counted in each section. An average of $10 \%$ or more tumor cells staining was defined as positive. Immunohistochemical staining was interpreted independently by two observers (L. H. X and L. Y. Q). The reproducibility was $95.5 \%(63 / 66)$. The cases with disagreement were discussed by the 2 observers and categorized according to consensus.

2.3. Statistical Analyses. All data were analyzed with SPSS version 11.0 statistical package (Shengce Software Ltd., Beijing, China). The differences in positive rates were analyzed

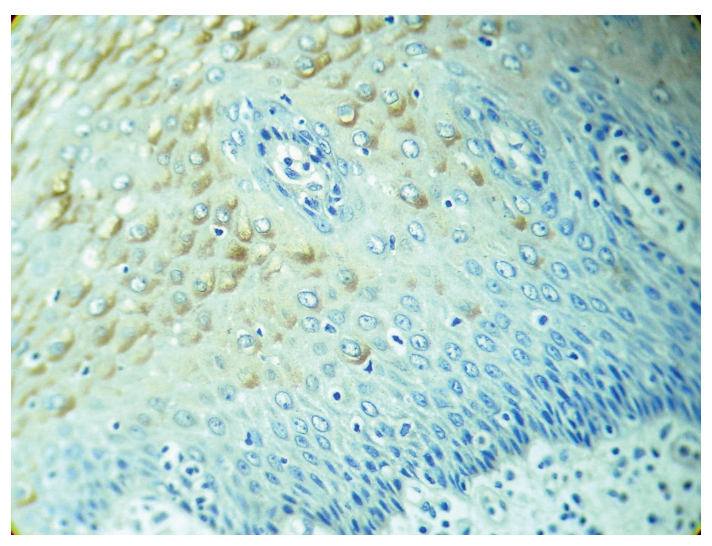

FIGURE 1: Immunoreactivity of PRL-1 protein in the normal esophageal epithelium $(\times 100)$.

TABLE 1: Frequency of PRL-1 protein expression in benign and malignant esophageal tissues.

\begin{tabular}{lccc}
\hline & Number of cases & PRL-1+ & $P$ values \\
\hline Normal & 20 & 0 & $<.001^{*}$ \\
esophagus & 60 & $41(68.3 \%)$ & \\
ESCC & 20 & $10(50.0 \%)$ & \\
$\quad$ ESCC (LN-) & 40 & $31(77.5 \%)$ & $0.031^{@}$ \\
$\quad$ ESCC (LN+) & 40 & $39(97.5 \%)$ & $0.003^{*}, 0.001^{@}$, \\
$\quad$ MESCC & 6 & $4(66.7 \%)$ & $0.025^{\#}$ \\
EA & & & $0.508^{*}$ \\
\hline
\end{tabular}

ESCC: esophageal squamous cell carcinoma; ESCC (LN-): ESCC without lymph node metastasis; ESCC (LN+): ESCC with lymph node metastasis; MESCC: metastatic ESCC; EA: esophageal adenocarcinoma; *: compared with ESCC; ${ }^{\circledR}$ : compared with ESCC (LN-); : compared with ESCC (LN+).

by $\chi^{2}$ test. The relationship of 2 variables was analyzed by correlation analysis. $P$ values less than .05 were defined as statistically significant.

\section{Results}

3.1. Expression of PRL-1 Protein in Benign Esophageal Tissue. PRL-1 immunoreactivity was visualized as brown granular staining in the cytoplasm. In normal esophageal mucosa, the staining was located only in the stratum spinosum and above, but not in the basal cell layers (Figure 1). Therefore, the staining of basal cell layers is used hereafter as index of PRL-1 expression in benign esophageal tissue. PRL-1 protein was also expressed in the skeletal muscle, ductal epithelia of esophageal glands, and plasma cells.

\subsection{Expression of PRL-1 Protein in Esophageal Neoplasm.} PRL-1 also showed cytoplasmic brown granular staining in ESCC, especially in tumor cells with keratin pearl (Figures 2 (a) and 2(b)). PRL-1 protein was expressed in $68.3 \%$ $(41 / 60)$ of ESCC, but not in any of normal esophageal mucosa $(P<.001$, Table 1$)$. 


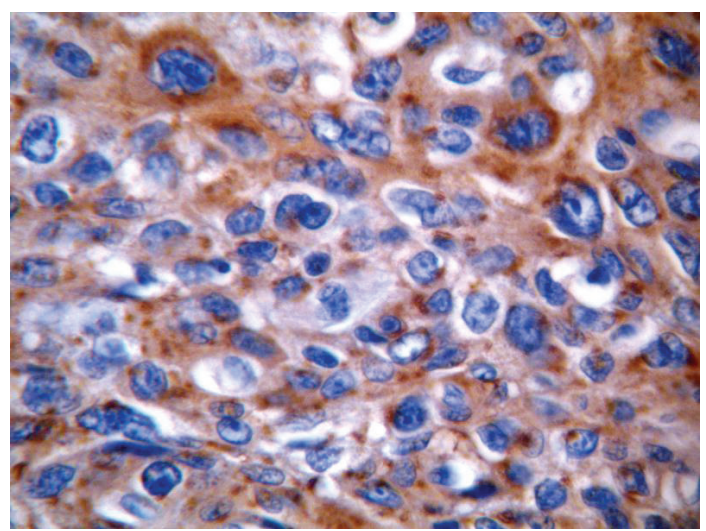

(a)

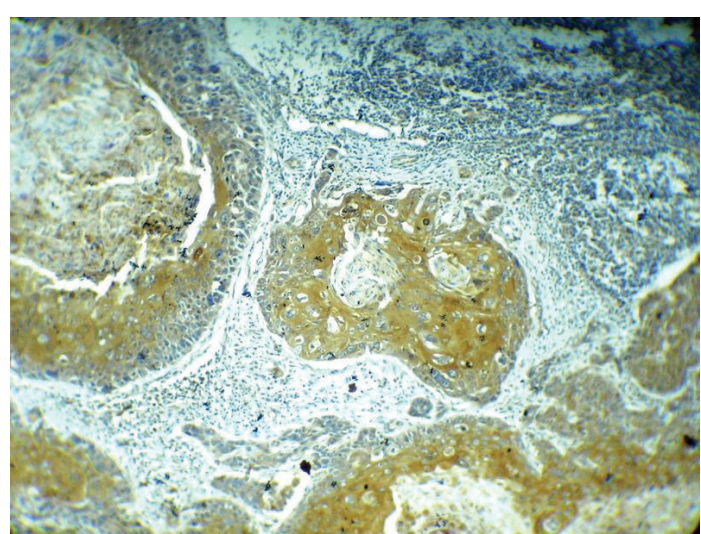

(b)

FIGURE 2: Immunoreactivity of PRL-1 protein in esophageal squamous cell carcinoma (ESCC). (a) In poorly differentiated ESCC $(\times 400)$; (b) in well differentiated ESCC, $\times 100$.

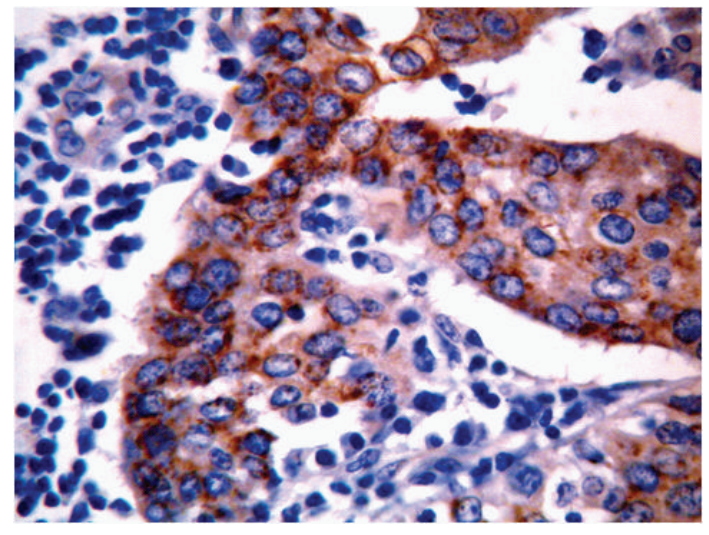

Figure 3: Immunoreactivity of PRL-1 protein in metastatic esophageal squamous cell carcinoma in lymph node $(\times 400)$.

To explore the association of PRL-1 protein with tumor metastasis, we compared the frequency of PRL-1 protein expressions in primary ESCC with and without lymph node metastasis. The results showed that PRL-1 protein was expressed in $77.5 \%$ (31/40) of ESCC with lymph node metastasis (Figure 3), significantly higher than that $(50 \%, 10 / 20)$ of ESCC without lymph node metastasis $(P=.031$, Table 1$)$.

To extend such exploration, we compared the frequencies of PRL-1 protein expression in metastatic ESCC with those in primary ESCC. The results showed that PRL-1 protein was expressed in $97.5 \%$ (39/40) of the metastatic ESCC in lymph nodes, significantly higher than that of the primary ESCC in every category $(77.5 \%(31 / 40))$ in the primary ESCC with lymph node metastasis $(P=.025) ; 50 \%(10 / 20)$ in the primary ESCC without lymph node metastasis $(P=.001)$; $68.3 \%(41 / 60)$ in all of 60 primary ESCCs $(P=.003$, Table 1$)$.

Out of 6 cases of esophageal adenocarcinoma (EA), PRL1 protein was expressed in 4 cases $(66.7 \%$, Table 1$)$. This frequency is similar to that of $\operatorname{ESCC}(P=.508$, Table 1$)$.
Interestingly, PRL-1 showed nuclear, in addition to cytoplasmic (Figure 4(a)), staining of tumor cells in 3 poorly differentiated EA (Figure 4(b)). The remaining one positive case was well-differentiated EA, showing solely cytoplasmic staining.

To examine the association of PRL-1 protein expression with clinicopathological factors, we compared its frequency between the patients' genders and ages, tumor's location, tumor differentiation, and stages. The results showed that the frequency of PRL-1 protein expression was $79.4 \%$ in stage III ESCC, significantly higher than that in stage 1 ESCC $(33.3 \%$, $P=.003$, Table 2), but similar to that in stage IIA (well, $76.5 \%, P=.12)$ and stage IIB $(66.7 \%, P=.491)$ ESCC. PRL-1 protein tends to express more often in well- and moderately differentiated ESCC than in poorly differentiated ESCC, but such differences were not significant $(P=.109$, $P=.069$, Table 2). Statistical analyses showed no significant association of PRL-1 protein expression in ESCC with the patients' sex and age or tumor size (Table 2).

\section{Discussion}

Since it was discovered in 1996 [8], PRL has drawn many researchers' attention due to its close association with human cancer and tumor metastasis. Experiments have shown that PRL can promote cell proliferation, enhance cell mobility, and induce metastatic tumor formation [10, 28]. PRL-3 expression has been extensively studied in a variety of tumor cell lines [12-14, 29, 30] and human cancers including colorectal, gastric, hepatic, ovarian, cholangiocarcinoma, nasopharyngeal, esophageal, and breast cancers [13-16, 18-23]. This study investigated the expression of $\mathrm{PRL}-1$ protein, another member of PRL family, and explored its association with metastasis of ESCC.

The expression of PRL-1 proteins in the normal esophageal epithelia was interestingly limited to differentiated squamous epithelia (stratum spinosum and above) with exclusion of the basal cell layers, the stem cells of esophageal epithelia. These results support the hypothesis that PRL is normally associated with terminal differentiation [25]. 


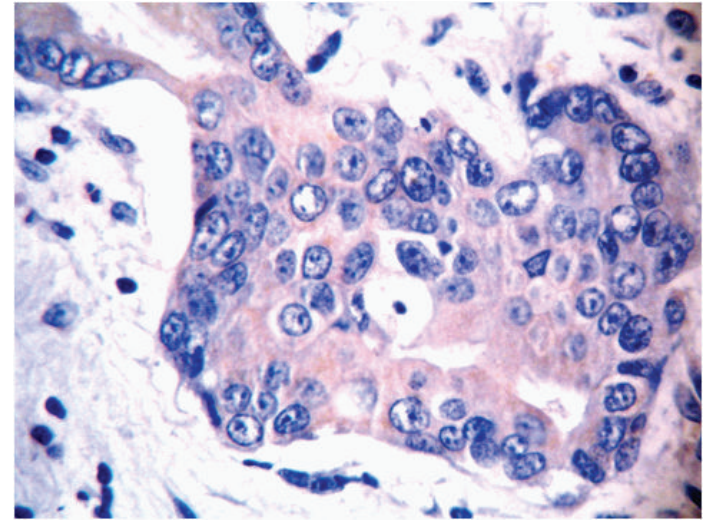

(a)

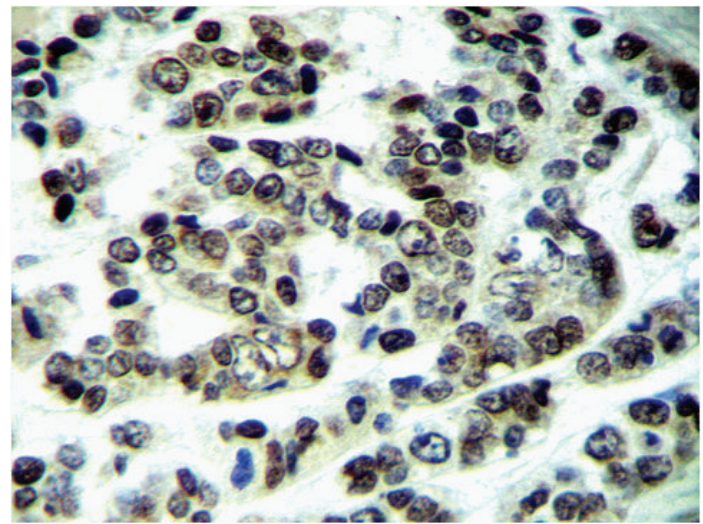

(b)

FIGURE 4: Immunoreactivity of PRL-1 protein in esophageal adenocarcinoma (EA). (a) Cytoplasmic staining in well-differentiated EA $(\times 400)$; (b) nuclear staining in poorly differentiated EA $(\times 400)$.

TABLE 2: Association of PRL-1 protein expression frequency with clinicopathological parameters.

\begin{tabular}{|c|c|c|c|}
\hline & Case $^{\#}$ & PRL-1+ & $P$ values \\
\hline \multicolumn{4}{|l|}{ Stages } \\
\hline I & 3 & $1(33.3 \%)$ & \\
\hline IIA & 17 & $10(58.8 \%)$ & .06 \\
\hline IIB & 6 & $4(66.7 \%)$ & $.058, .735^{*}$ \\
\hline III & 34 & $27(79.4 \%)$ & $.003, .12^{*}, .491^{\#}$ \\
\hline \multicolumn{4}{|c|}{ Tumor differentiation } \\
\hline Well & 11 & $9(81.8 \%)$ & \\
\hline Moderate & 34 & $25(73.5 \%)$ & .705 \\
\hline Poorly & 15 & $7(46.7 \%)$ & $.109, .069^{*}$ \\
\hline \multicolumn{4}{|c|}{ Tumor location } \\
\hline Upper $1 / 3$ & 12 & $8(66.7 \%)$ & \\
\hline Mid $1 / 3$ & 39 & $28(71.8 \%)$ & .733 \\
\hline Lower $1 / 3$ & 8 & $5(55.6 \%)$ & $.604, .343^{*}$ \\
\hline \multicolumn{4}{|c|}{ Patients' gender } \\
\hline Male & 38 & $27(71.1 \%)$ & \\
\hline Female & 22 & $14(63.6 \%)$ & .552 \\
\hline \multicolumn{4}{|l|}{ Patients' age } \\
\hline$\leq 60$ yrs & 32 & $22(68.8 \%)$ & \\
\hline$>60$ yrs & 28 & $19(67.9 \%)$ & .941 \\
\hline
\end{tabular}

* compared with the second group; ${ }^{*}$ compared with the third group.

The similar expression pattern is also seen in the expression of other molecules including CK20. CK20 is expressed only in the top layer, umbrella cells, of normal urothelial epithelial cells [31], and such pattern has been widely used by surgical pathologists to differentiate neoplastic lesion from reactive process in the urinary bladder. Therefore, lack of PRL-1 staining in the basal cell layers was defined as normal in nonneoplastic esophageal epithelia in this study. Based on these criteria, PRL-1 protein was not expressed in paired normal esophageal mucosa $(0 \%)$ in contrast to $68.3 \%$ in primary ESCC $(P<.01)$. This frequency is similar to that (78\%) of PRL-3 in ESCC [22]. PRL-3 was reported to closely associate with tumor's metastasis in human cancers including colorectal, gastric, and breast cancers $[16,17,32-$ 35]. In this study, the frequency of PRL-1 protein expression was significantly higher in ESCC with lymph node metastasis than that in ESCC without lymph node metastasis and also significantly higher in metastatic ESCC in lymph node than that in primary ESCC. Our results are consistent with those reported for other human cancers and suggest that PRL-1 may be involved in promoting the metastasis of ESCC.

PRL-1 protein was also expressed in the majority (3/4, $66.7 \%$ ) of esophageal adenocarcinoma (EA), similar to its expression frequency in ESCC $(68.3 \%, 41 / 60)$. PRL-1 was also interestingly expressed in the nuclei, in addition to the cytoplasm, of tumor cells in the poorly differentiated EA, but not in any ESCC. This characteristic nuclear staining of PRL-1 in EA suggests that PRL-1 may have broader clinical implication than PRL-3 since nuclear staining has not been reported for PRL-3. Presence of nuclear staining will facilitate differential diagnosis of poorly differentiated EA from poorly differentiated ESCC. Such differential diagnosis remains a big challenge for the surgical pathologist, especially when tumor is located in the lower $1 / 3$ of the esophagus. The clinical application of PRL-1's nuclear staining needs to be further explored with more cases. The mechanism of due nuclear and cytoplasmic staining of PRL-1 is not clear. It may be associated with its function and molecule trafficking within cells. PRL-1 was originally thought as a nuclear protein [10], but subsequent study revealed that all three PRLs are mainly associated with the membrane structure [36]. The subcellular localization of endogenous PRL exhibited cell cycledependent pattern as shown with immunofluorescence. In nonmitotic HeLa cells, PRL-1 was present in the endoplasmic reticulum in a farnesylation-dependent manner. However, in mitotic cells, it relocalized to the centrosomes and the spindle apparatus in a farnesylation-independent manner [12]. PRL1 was consistently expressed in the nuclei of mature cells with the development progression [25]. 
Studies have shown that expression of PRL-3 is positively correlated with the stage of colorectal, gastric, and ovarian cancers $[15,16,35-37]$ and reversely correlated with the disease-free survival in breast cancer [34]. In addition, PRL3 expression was also closely associated with tumor size, lymphatic and venous invasion, and extent of lymph node metastasis in gastric cancer [35]. Ooki et al. reported that, in ESCC, PRL-3 was independent prognostic factor for LMN stage, not associated with any other clincopathological parameter [22]. Our results showed the similar association for PRL-1 protein in ESCC and indicated that PRL-1 may serve as a good biomarker to predict the aggressiveness, especially the potential of metastasis, of ESCC.

Overexpression of PRL-3 in metastatic tumor has intrigued people to explore its potential role as the therapeutic target for cancers. The effectiveness and feasibility of such approach have been proved experimentally. The expression of the catalytically inactive PRL-3 mutant significantly reduced the cell migratory capability [14]. The higher migratory ability in PRL-3-overexpressed cells could be reversed by specific antisense oligodeoxynucleotide and the phosphatase inhibitors [14]. PRL-3-specific knockdown using small interfering RNA severely impaired the growth of ovarian cancer cells [15] and abrogated motility (in vitro) and hepatic colonization (in vivo) of human colon cancer DLD-1 cells [17]. The antiprotozoa drug, pentamidine, is an inhibitor of PRLs and can inactivate exogenous PRLs with a long effective duration $(>24 \mathrm{~h})$ after a pulse cell treatment at its therapeutic dose. It also inhibits growth in human cancer cell lines expressing endogenous PRLs. The growth of WM9 human melanoma tumors could also be markedly inhibited by pentamidine at a tolerable dose in nude mice with the induction of tumor cell necrosis [38]. Extremely high frequency of PRL-1 protein expression in primary ESCC (up to $68.3 \%$ ) and their metastatic lesions (up to 97.5\%) in lymph nodes identified in this study will provide further evidence for exploring such possibility to prevent and treat metastasis of ESCC in the future.

\section{Acknowledgment}

Y. Liu and X. Lou authors contribuate equally.

\section{References}

[1] U. J. Ribeiro, M. Posner, R. A. Safatle et al., "Risk factors for squamous cell carcinoma of the oesophagus," British Journal of Surgery, vol. 83, no. 9, pp. 1174-1185, 1996.

[2] L. D. Li and F. Z. Lu, "Survey on the death of malignant tumors in China," China Cancer, vol. 5, pp. 3-7, 1996.

[3] H. E. Gabbert, T. Shimoda, P. Hainaut et al., "Squamous cell carcinoma of the esophagus," in Tumors of the Digestive System, S. R. Hamilton and L. A. Aaltonen, Eds., pp. 11-19, IARC Press, Lyon, France, 2000, World Health Organization Classification of Tumors.

[4] X. Chen and C. S. Yang, "Esophageal adenocarcinoma: a review and perspectives on the mechanism of carcinogenesis and chemoprevention," Carcinogenesis, vol. 22, no. 8, pp. 1119-1129, 2001.
[5] T. Hunter, "Signaling—2000 and beyond," Cell, vol. 100, no. 1, pp. 113-127, 2000.

[6] M. A. Lyon, A. P. Ducruet, P. Wipf et al., "Dual-specificity phosphatases as targets for antineoplastic agents," Nature Reviews Drug Discovery, vol. 1, no. 12, pp. 961-976, 2002.

[7] B. T. Hoffman, M. R. Nelson, K. Burdick et al., "Protein tyrosine phosphatases: strategies for distinguishing proteins in a family containing multiple drug targets and anti-targets," Current Pharmaceutical Design, vol. 10, no. 10, pp. 1161-1181, 2004.

[8] C. A. Cates, R. L. Michael, K. R. Stayrook et al., "Prenylation of oncogenic human $\mathrm{PTP}(\mathrm{CAAX})$ protein tyrosine phosphatases," Cancer Letters, vol. 110, no. 1-2, pp. 49-55, 1996.

[9] Q. Zeng, W. Hong, and Y. H. Tan, "Mouse PRL-2 and PRL3 , two potentially prenylated protein tyrosine phosphatases homologous to PRL-1," Biochemical and Biophysical Research Communications, vol. 244, no. 2, pp. 421-427, 1998.

[10] R. H. Diamond, D. E. Cressman, T. M. Laz et al., "PRL-1, a unique nuclear protein tyrosine phosphatase, affects cell growth," Molecular and Cellular Biology, vol. 14, no. 6, pp. 3752-3762, 1994.

[11] W. F. Matter, T. Estridge, C. Zhang et al., "Role of PRL-3, a human muscle-specific tyrosine phosphatase, in angiotensinII signaling," Biochemical and Biophysical Research Communications, vol. 283, no. 5, pp. 1061-1068, 2001.

[12] J. Wang, C. E. Kirby, and R. Herbst, "The tyrosine phosphatase PRL-1 localizes to the endoplasmic reticulum and the mitotic spindle and is required for normal mitosis," Journal of Biological Chemistry, vol. 277, no. 48, pp. 46659-46668, 2002.

[13] U. A. Miskad, S. Semba, H. Kato et al., "Expression of PRL3 phosphatase in human gastric carcinomas: close correlation with invasion and metastasis," Pathobiology, vol. 71, no. 4, pp. 176-184, 2004.

[14] X. Wu, H. Zeng, X. Zhang et al., "Phosphatase of regenerating liver-3 promotes motility and metastasis of mouse melanoma cells," American Journal of Pathology, vol. 164, no. 6, pp. 20392054, 2004.

[15] F. Polato, A. Codegoni, R. Fruscio et al., "PRL-3 phosphatase is implicated in ovarian cancer growth," Clinical Cancer Research, vol. 11, no. 19 I, pp. 6835-6839, 2005.

[16] G. P. Zhao, Z. G. Zhou, W. Z. Lei et al., "Expression of phosphatase of regenerating liver-3 mRNA and its clinical implications in human colorectal carcinoma," Zhonghua Wei Chang Wai Ke Za Zhi, vol. 8, no. 3, pp. 237-240, 2005.

[17] H. Kato, S. Semba, U. A. Miskad et al., "High expression of PRL-3 promotes cancer cell motility and liver metastasis in human colorectal cancer: a predictive molecular marker of metachronous liver and lung metastases," Clinical Cancer Research, vol. 10, no. 21, pp. 7318-7328, 2004.

[18] T. Ren, B. Jiang, X. Xing et al., "Prognostic significance of phosphatase of regenerating liver-3 expression in ovarian cancer," Pathology and Oncology Research, vol. 15, no. 4, pp. 555-560, 2009.

[19] N. Dai, A. P. Lu, C. C. Shou et al., "Expression of phosphatase regenerating liver 3 is an independent prognostic indicator for gastric cancer," World Journal of Gastroenterology, vol. 15, no. 12, pp. 1499-1505, 2009.

[20] Y. Xu, M. Zhu, S. Zhang et al., "Expression and prognostic value of PRL-3 in human intrahepatic cholangiocarcinoma," Pathology and Oncology Research, vol. 16, no. 2, pp. 169-175, 2010.

[21] J. Zhou, S. Wang, J. Lu et al., "Over-expression of phosphatase of regenerating liver- 3 correlates with tumor progression and 
poor prognosis in nasopharyngeal carcinoma," International Journal of Cancer, vol. 124, no. 8, pp. 1879-1886, 2009.

[22] A. Ooki, K. Yamashita, S. Kikuchi et al., "Phosphatase of regenerating liver-3 as a prognostic biomarker in histologically node-negative gastric cancer," Oncology Reports, vol. 21, no. 6, pp. 1467-1475, 2009.

[23] R. T. Hao, X. H. Zhang, Y. F. Pan et al., "Prognostic and metastatic value of phosphatase of regenerating liver-3 in invasive breast cancer," Journal of Cancer Research and Clinical Oncology, vol. 136, no. 9, pp. 1349-1357, 2010.

[24] Y. Q. Liu, H. X. Li, X. Lou et al., "Expression of phosphatase of regenerating liver 1 and 3 mRNA in esophageal squamous cell carcinoma," Archives of Pathology and Laboratory Medicine, vol. 132, no. 8, pp. 1307-1312, 2008.

[25] W. Kong, G. P. Swain, S. Li et al., "PRL-1 PTPase expression is developmentally regulated with tissue-specific patterns in epithelial tissues," American Journal of Physiology, vol. 279, no. 3, pp. G613-G621, 2000.

[26] J. Li, K. Guo, V. W Koh et al., "Generation of PRL-3- and PRL-1-specific monoclonal antibodies as potential diagnostic markers for cancer metastases," Clinical Cancer Research, vol. 11, no. 6, pp. 2195-2204, 2005.

[27] L. Peng, J. Ning, L. Meng et al., "The association of the expression level of protein tyrosine phosphatase PRL-3 protein with liver metastasis and prognosis of patients with colorectal cancer," Journal of Cancer Research and Clinical Oncology, vol. 130, no. 9, pp. 521-526, 2004.

[28] Q. Zeng, J. M. Dong, K. Guo et al., "PRL-3 and PRL-1 promote cell migration, invasion, and metastasis," Cancer Research, vol. 63, no. 11, pp. 2716-2722, 2003.

[29] H. Han, D. J. Bearss, L. W. Browne et al., "Identification of differentially expressed genes in pancreatic cancer cells using cDNA microarray," Cancer Research, vol. 62, no. 10, pp. 28902896, 2002.

[30] Q. Wang, D. I. Holmes, S. M. Powell et al., "Analysis of stromal-epithelial interactions in prostate cancer identifies PTPCAAX2 as a potential oncogene," Cancer Letters, vol. 175, no. 1, pp. 63-69, 2002.

[31] P. Harnden, I. Eardley, A. D. Joyce et al., "Cytokeratin 20 as an objective marker of urothelial dysplasia," British Journal of Urology, vol. 78, no. 6, pp. 870-875, 1996.

[32] S. Saha, A. Bardelli, P. Buckhaults et al., "A phosphatase associated with metastasis of colorectal cancer," Science, vol. 294, no. 5545, pp. 1343-1346, 2001.

[33] A. Bardelli, S. Saha, J. A. Sager et al., "PRL-3 expression in metastatic cancers," Clinical Cancer Research, vol. 9, no. 15, pp. 5607-5615, 2003.

[34] I. Radke, M. Götte, C. Kersting et al., "Expression and prognostic impact of the protein tyrosine phosphatases PRL-1, PRL-2, and PRL-3 in breast cancer," British Journal of Cancer, vol. 95, no. 3, pp. 347-354, 2006.

[35] U. A. Miskad, S. Semba, S. Kato et al., "High PRL-3 expression in human gastric cancer is a marker of metastasis and grades of malignancies: an in situ hybridization study," Virchows Archiv, vol. 450, no. 3, pp. 303-310, 2007.

[36] Q. Zeng, X. Si, H. Horstmann et al., "Prenylation-dependent association of protein-tyrosine phosphatases PRL-1, -2, and -3 with the plasma membrane and the early endosome," Journal of Biological Chemistry, vol. 275, no. 28, pp. 21444-21452, 2000.

[37] T. E. Buffart, J. Coffa, M. A. Hermsen et al., "DNA copy number changes at 8q11-24 in metastasized colorectal cancer," Cellular Oncology, vol. 27, no. 1, pp. 57-65, 2005.
[38] M. K. Pathak, D. Dhawan, D. J. Lindner et al., "Pentamidine is an inhibitor of PRL phosphatases with anticancer activity," Molecular Cancer Therapeutics, vol. 1, no. 14, pp. 1255-1264, 2002. 


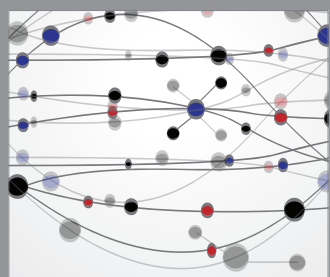

The Scientific World Journal
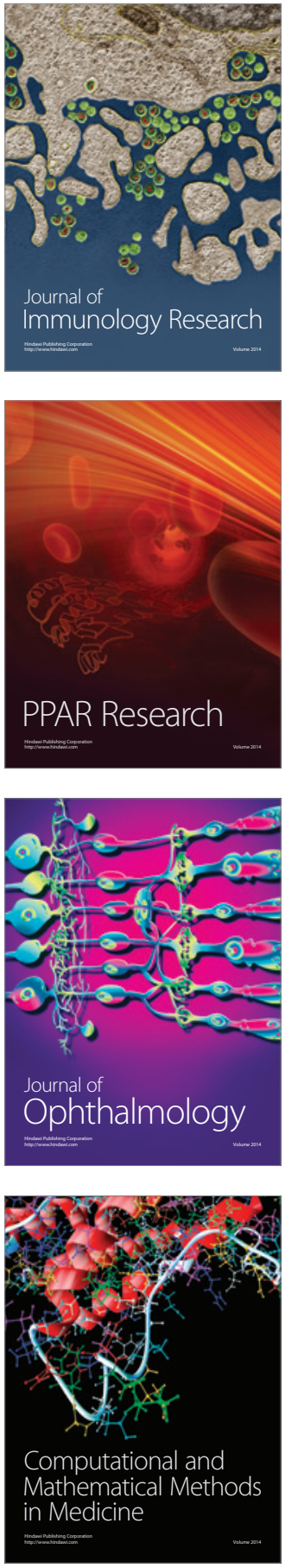

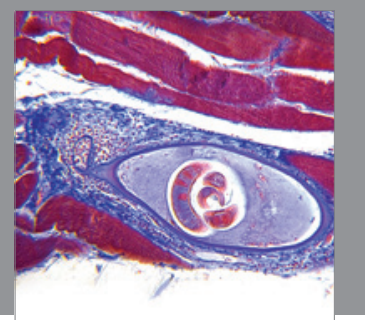

Gastroenterology

Research and Practice
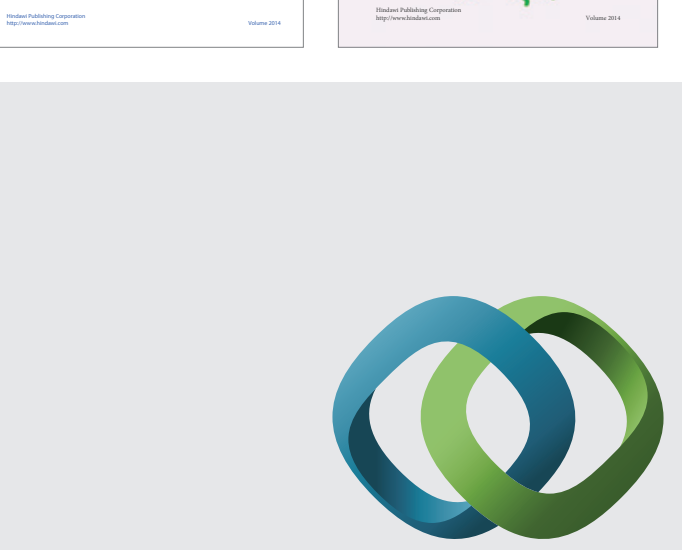

\section{Hindawi}

Submit your manuscripts at

http://www.hindawi.com
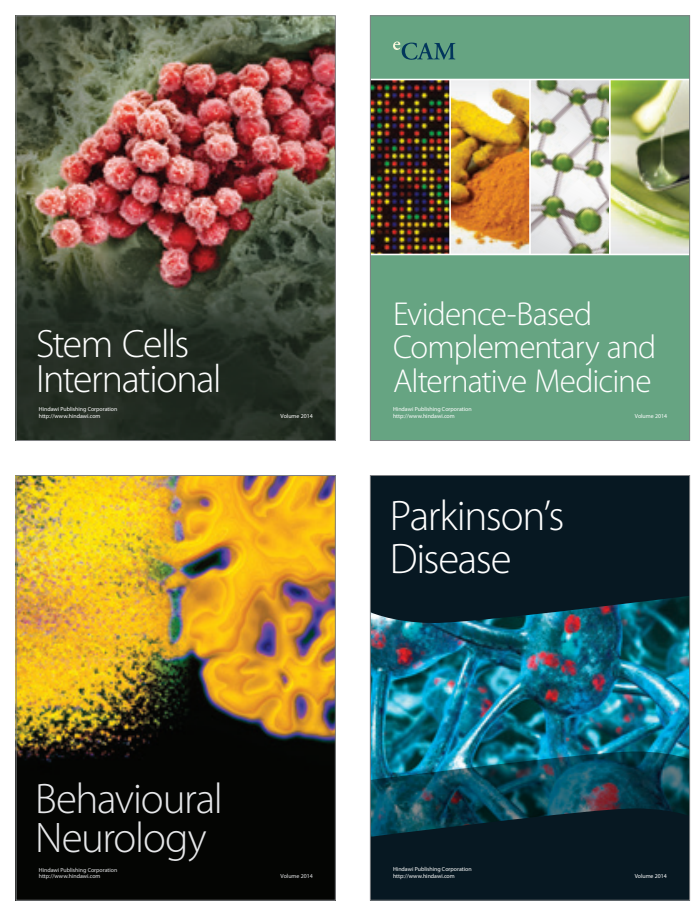

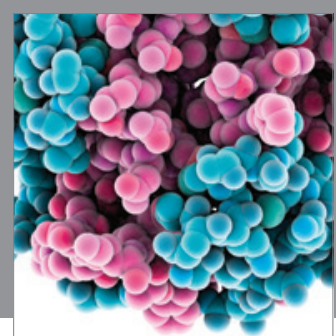

Journal of
Diabetes Research

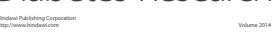

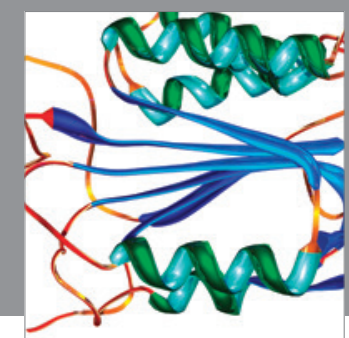

Disease Markers
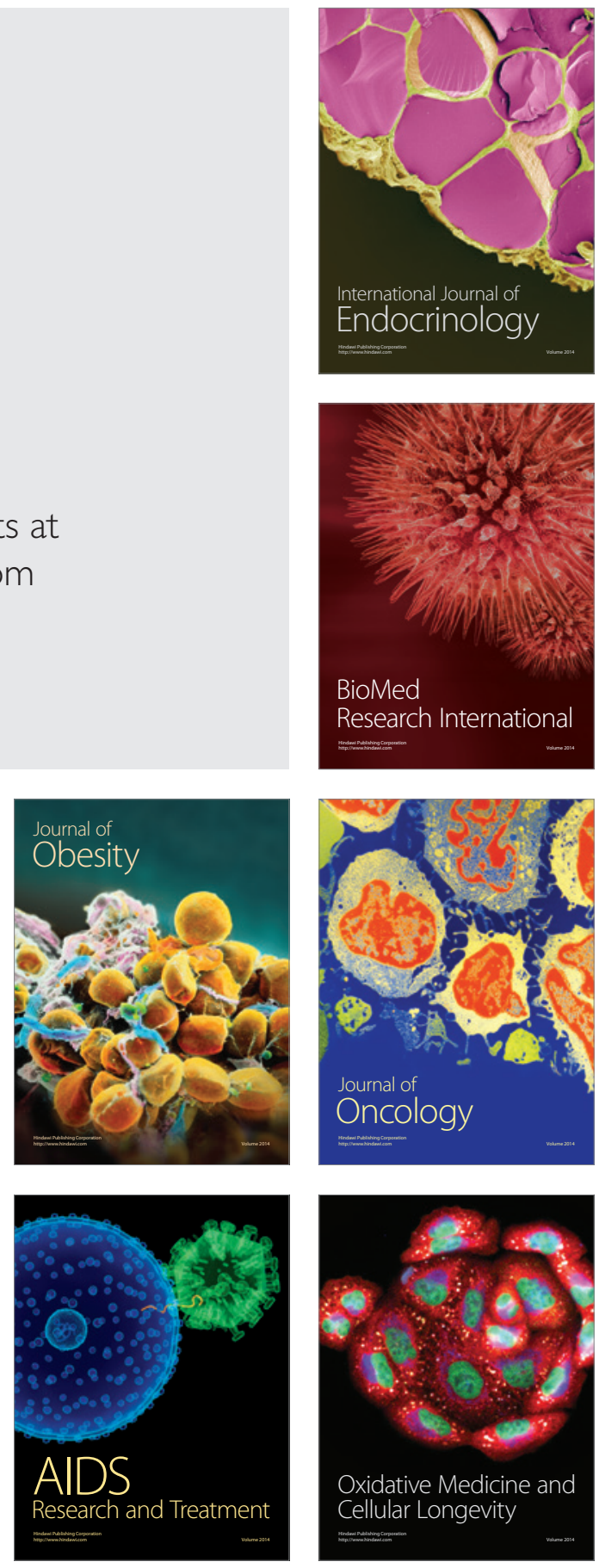